\title{
Trombosis "extremadamente" tardía de stent
}

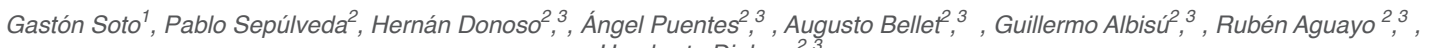
Humberto Dighero ${ }^{2}, 3$.

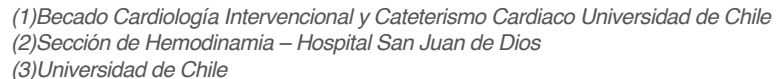

\section{Introducción}

La angioplastía coronaria constituye actualmente el tratamiento recomendado en síndrome coronario agudo con elevación del segmento ST ${ }^{1,2}$. A pesar de ello la angioplastía se encuentra frente a dos problemas relevantes: restenosis y trombosis. La primera ha sido considerada históricamente como el problema más importante en la Cardiología Intervencionista ${ }^{3}$, siendo la estrategia terapéutica que más ha incidido en su disminución (hasta un $6 \%$ en la actualidad) la utilización de stent medicados (Drug Eluting Stent - DES ${ }^{4}$

La trombosis de stent (TS) es un fenómeno menos frecuente que la restenosis $(<1 \%)$, pero dramático debido a su elevada mortalidad $(20-40 \%)$ e infarto al miocardio $(50-70 \%)^{5,6,7}$. La fisiopatología de la TS es multifactorial; la literatura indica una serie de elementos que pueden actuar solos o en combinación, entre lo que encontramos factores relacionados con el procedimiento (incluidos aquellos originados en el stent) y otros producidos en el paciente ${ }^{8,9}$

La clasificación de la trombosis de stent fue determinada por la ARC (Academic Research Consortium) en donde se destaca además de la "probabilidad" de su

Correspondencia:

Dr. Gastón Soto Espinoza

Sección de Hemodinamia - Hospital San Juan de Dios

Huérfanos 3255 - Santiago

gastonsotoe@gmail.com presentación (definitiva - probable o posible), la relación temporal de la presentación a la implantación de stent. Esta la divide en precoz (menos de 30 días), temprana (entre 30 días y 1 año) y tardía (mayor a 1 año) ${ }^{10}$. La importancia de esta clasificación radica en que se puede establecer un vínculo entre la temporalidad de la presentación de la trombosis de stent y la fisiopatología de esta. ${ }^{11}$

Las guías clínicas señalan que aquellos pacientes que reciben DES en su terapéutica deberían mantener la terapia antiplaquetaria dual por un mínimo de 12 meses $^{12}$. En los distintos subtipos de trombosis de stent (en relación a su presentación temporal) sea esta precoz, temprana o tardía, ésta última corresponde a la de menor frecuencia. Debido a lo anterior a continuación describimos el caso clínico de un paciente que presentó TS medicado que podríamos denominar "extremadamente" tardía (4 años post implante), cuya evolución fue favorable.

\section{Caso clínico}

Paciente de 56 años, con antecedentes de HTA $1^{\circ}$ (tratamiento enalapril), DM tipo 2 no insulino requirente (tratamiento metformina) y síndrome coronario 

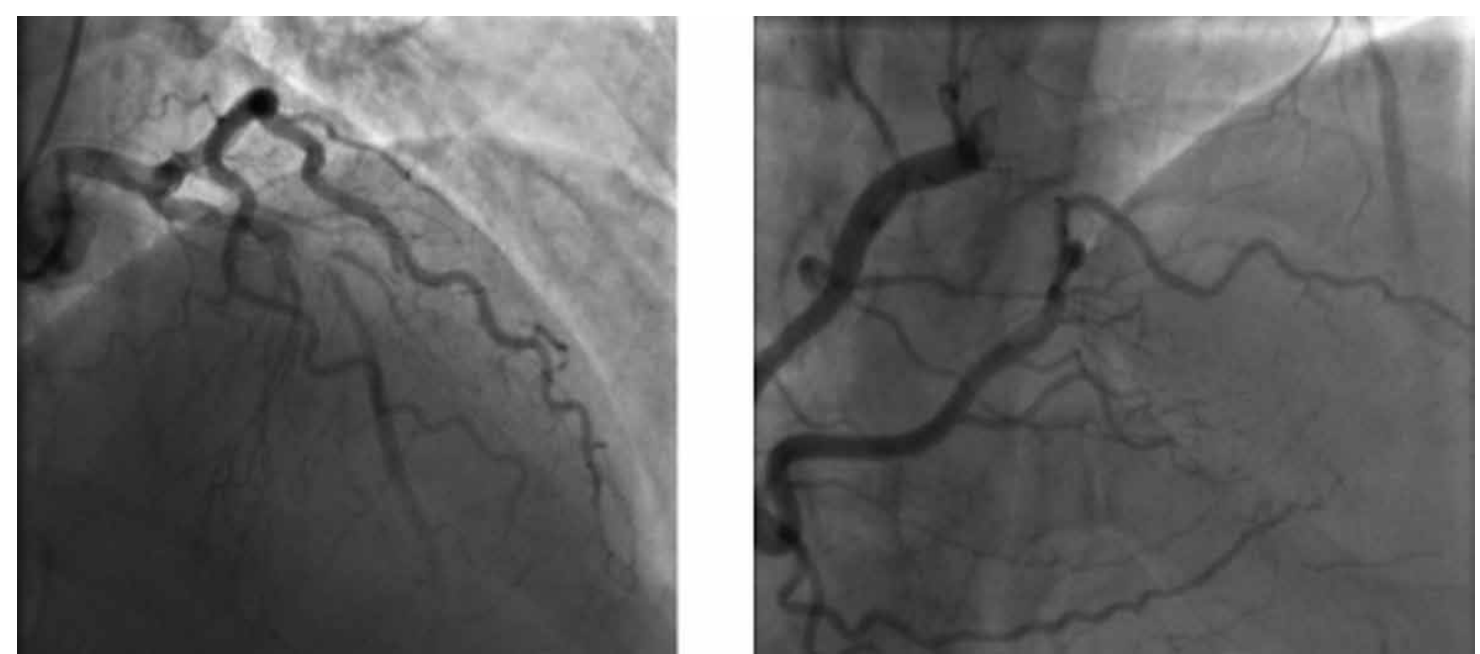

Figura 1. Coronariografía que concluye; ADA: vaso de buen desarrollo, flujo distal TIMI II. Presenta una placa proximal excéntrica, ¿Bicelada?, que disminuye su calibre en un 50\%, inmediatamente después presenta otra lesión larga con imagen de trombo que compromete el origen de un ramo diagonal de pequeño calibre en un $90 \%$ (largo $21.7 \mathrm{~mm}$ ), resto del vaso sin estenosis. ACD: sin estenosis angiográficas da circulación colateral a izquierda. Resto de los vasos sin lesiones angiográficas.

agudo (prevención $2^{\circ}$ con aspirina, atorvastatina , atenolol). Presenta consulta inicial en Unidad Coronaria (UCO) en Hospital San Juan de Dios (HSJD) en junio del 2007 por angina inestable. Se solicita estudio angiográfico (score TIMI 3, GRACE 47). (Fig1). Este concluye: lesión de 50\% en segmento proximal de arteria descendente anterior (ADA) que continúa con imagen de trombo que compromete ramo diagonal. La arteria coronaria derecha (ACD) otorga circulación colateral a ADA (Clasificación
Rentrop - Arai I o o Elayda grupo I) .

Debido a lo anterior se decide angioplastía ad hoc de esta lesión con stent Firebird ${ }^{\circledR}$ 3.5x33mm (Fig 2.) . Paciente continúa controles habituales en policlínico de Cardiología (a terapéutica farmacológica antes señalada se agrega clopidogrel por 1 año) .

En febrero 2011 consulta en atención primaria por SCA s/SDST anterior, nuevamente se solicita angiografía coronaria (Fig 3.) que concluye arterias coronarias sin lesiones angiográficas significativas.

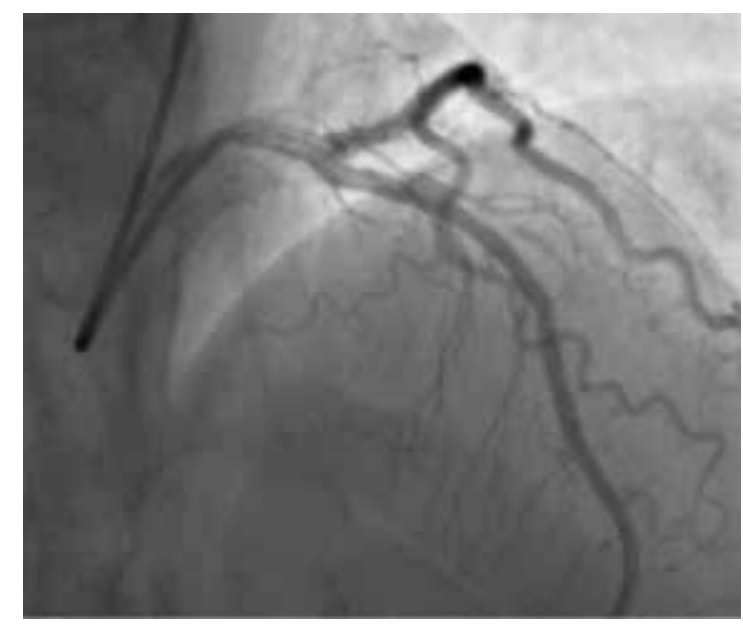

Figura 2. Angioplastía de lesión con stent FIREBIRD ${ }^{\circledR} 3.5 \times 33$. Flujo TIMI III post procedimiento.

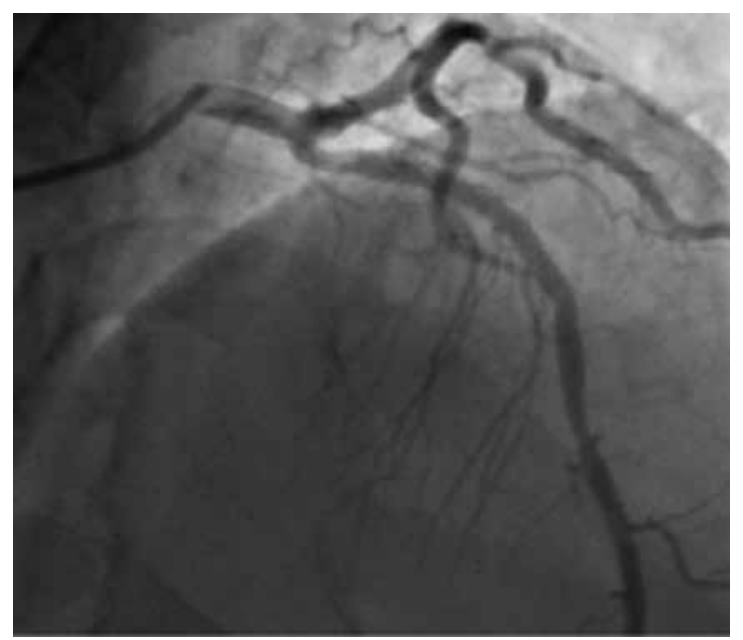

Figura 3. Arterias coronarias sin lesiones angiográficas significativas. Stent a ADA sin restenosis. 
Último ingreso en UCO de HSJD en octubre de 2011 por angina inestable de riesgo moderado (score TIMI 4), en este oportunidad la angiografía coronaria (Fig 4.) concluye : stent en segmento proximal de ADA ocluido , este vaso se llena por colaterales heterocoronarias ( Rentrop - Arai II ). Resto de los vasos sin lesiones angiográficas significativas.
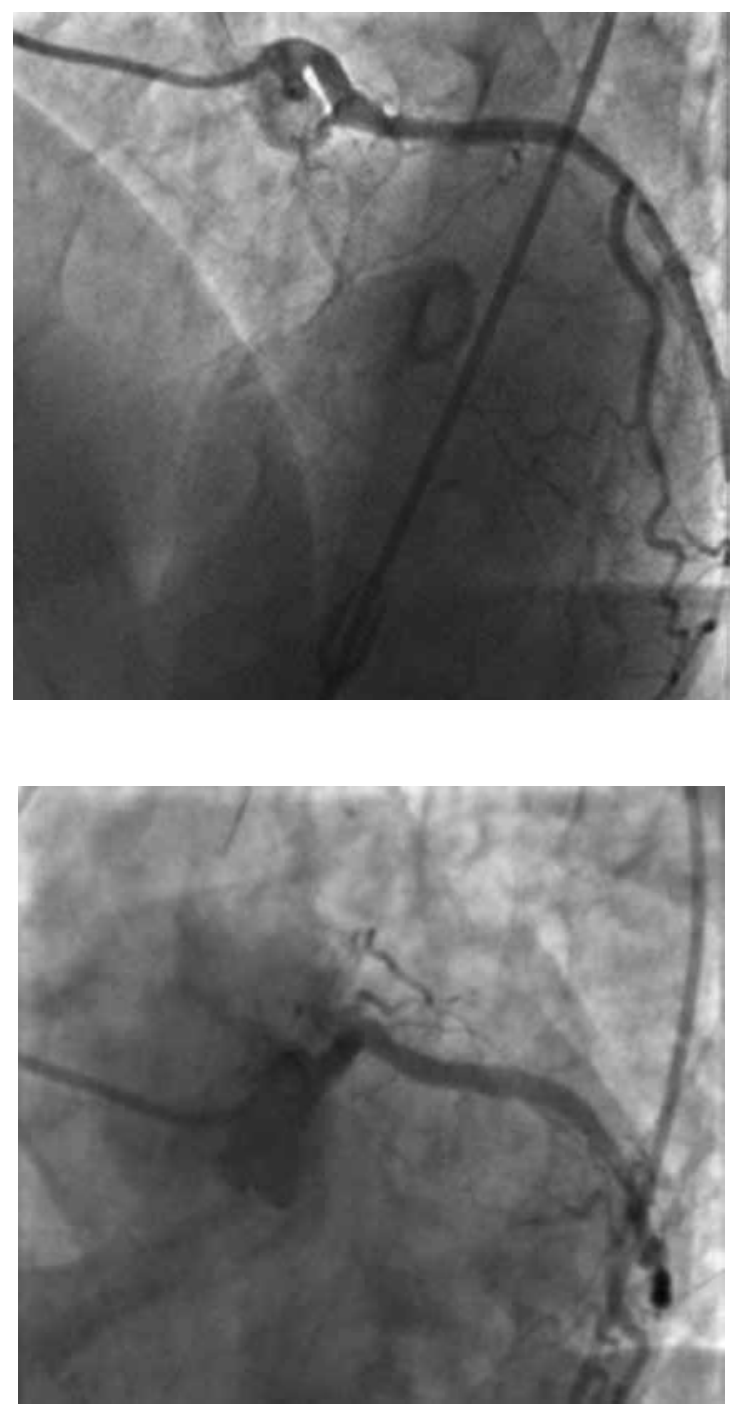

La lesión previamente señalada es sugerente de trombosis muy tardía de stent .

\section{Discusión}

La trombosis de stent es un fenómeno de baja frecuencia $(<1 \%)(5)$, pero de elevada morbilidad ( IAM $50-70 \%$ de los pacientes ) y mortalidad (20-40\%) (5).

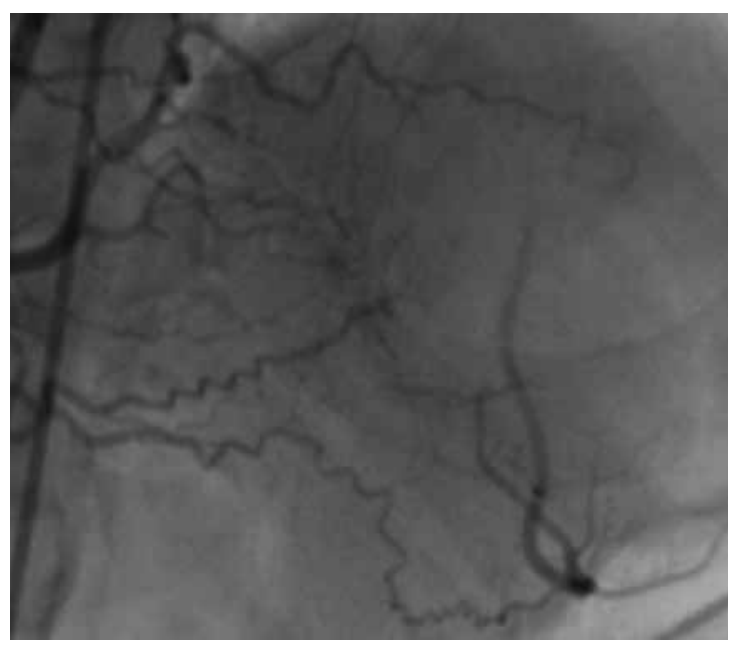

Figura 4. Coronariografía que concluye enfermedad coronaria severa de 1 vaso. Oclusión de stent a ADA sugerente de trombosis muy tardía de stent. Colaterales heterocoronarias a ADA.

La ARC (American Research Consortium) estableció las definiciones de TS (7) en relación a la posibilidad de presentar ésta (definitiva , probable y posible) y la temporalidad de ocurrencia (precoz , tardía o muy tardía)

Nuestro paciente cumple con dos criterios de esta clasificación. Primero es una TS definitiva (por la demostración angiográfica) y es muy tardía (ocurrió más de 1 año luego de su implantación el 2007).

La fisiopatología es múltiple. La TS precoz se relaciona a lesiones residuales que se trombosan, disecciones, estasis sanguíneo e infra expansión de stent ${ }^{13}$ A diferencia de ésta, la TS tardía y muy tardía se asocia a endotelización incompleta ${ }^{14} \mathrm{o}$ la presencia de placas ateromatosas de aparición muy tardía en el stent ${ }^{15}$

La ventaja de los stent liberadores de drogas (DES) en comparación con aquellos que no lo son (BMS: Bare Metal Stent) es la disminución de la reestenosis a menos de una $6 \%^{3}$. La desventaja es la necesidad de doble anti agregación por un período no inferior a 12 meses en el caso de los DES ${ }^{12}$ el cual supone un riesgo aumentado de hemorragia en un grupo de pacientes determinados 12 .

Nuestro paciente nos parece importante reportar por dos razones: en primer lugar la forma TS que presentó es la más infrecuente (trombosis muy tardía), la 
cual ocurrió 4 años post implante. En segundo lugar la forma de presentación fue rara (angina inestable) y el pronóstico favorable (recordemos mortalidad de 20-40\%).

Al revisar la evidencia de la terapia antiplaquetaria dual en intervencionismo coronario nos indican que aquellos pacientes que utilizan stent liberadores de drogas (tanto en síndrome coronario agudo, como en aquellos que no lo presentan) el uso de este debe ser por lo menos de 12 meses (como ocurrió en el paciente), la suspensión de ésta antes de este período puede ser muy arriesgado como lo señala la literatura ${ }^{16}$, 17, 18, 19. Lo ocurrido con nuestro paciente es inusual (trombosis muy tardía), pero ¿Existe evidencia para mantener la doble antiagregación por un período mayor a 12 meses?. La nuevas guías de intevencionismo coronario $^{12}$ señalan sólo esta indicación con nivel de evidencia $\mathrm{C}$ (recomendación de expertos) . 


\section{Bibliografía}

1. KEELEY EC , BOURA JA, GRIBES CL . Primary angioplasty versus intravenous thrombolytic therapy for acute myocardial infarction: a quantitative review of 23 randomised trials . Lancet $2003 ; 361: 13-20$.

2. GUARDA E, PRIETO JC, SANHUEZA P, DAUVERNE M, ASENJO R, CORBALAN R. Guías 2009 de la Sociedad Chilena de Cardiología para el tratamiento del Infarto Agudo al Miocardio con supradesnivel del ST . Revista Chilena de Cardiología 2009; 28: 229-230.

3. DANGAS G,BIMMER C,CAIXETAA,SANIDAS E, MINTZ G, MEHRAN R. In - stent restenosis in the drug - eluting stent era. J Am Coll Cardiol 2010; 56: 1897-1890.

4. STETTLER C, WANDEL S, ALLEMANN S. Outcomes associated with drug - eluting and bare -metal stents : a collacorative network meta - análisis . Lancet 2007; 370: 937-948.

5. HOLMES D, KERIAKEZ D, GARG S, SERRYUS P, DEHMER G, ELLIS S, et al . Stent thrombosis. 2010; 56: 1357- 1363.

6. IAKOVOU I, SCHMIDT T, BONIZZONI E. Incidence, predictors , and outcome of thrombosis after successful implantation of drug-eluting stents. JAMA 2005;293:2126-30.

7. CUTLIP DE, WINDECKER S, MEHRAN R, BOAM A, COHEN DJ, VAN ES GA, et al. Clinical end points in coronary stent trials : a case for standardized definitions. Circulation 2007; 115: 2344-51

8. MAURI L, HSIEH W, MASSARO J, HO KK, D'AGOSTINO $R$, CUTLIP D. Stent thrombosis in randomized clinical trials of drug-eluting stents. N Engl J Med 2007;356:1020-9.

9. SPAULDING C, DAEMEN J, BOERSMA E, CUTLIP D, SERRUYS P . A pooles analysis of data comparing sirolimus-eluting stents with bare metal stents . N Engl J Med 2007;356:98997.

10. CUTLIPC, WINDECKER S, MEHRAN R. Clinical end points in coronary stent trials: a case for standardized definitions . Circulation 2007;115:2344-51.

11. JONER M, FINN A; FARB A. Pathology of drug-eluting stents in humans: delayed healing and late thrombotic risk. J Am Coll Cardiol 2006; 48: 193-202 .

12. LEVINE GLENN N, BATES ERIC R, BLANKENSHIP JAMES C, BAILEY STEVEN R, BITTL JOHNA, CERCEK BOJAN, et al. 2011 ACCF/AHA/SCAI Guideline for Pecutaneous Coronary Intervention. 2011;58:32-34.

13. FUJII K, CARLIER SG, MINTZ GS, YANG YM, MOUSSA I, WEISZ G, et al. Stent underexpansion and residual reference segment stenosis ara related to stent thrombosis after sirolimuseluting stent implantation: an intravascular ultrasound study. J Am Coll Cardiol 2005;45:995-998 .

14. JONER M, FINN AV, FARB A, MONT EK, KOLODGIE FD, LADICH E, et al . Pathology of drug - eluting stents in humans: delayed healing and late thrombotic risk. J Am Coll Cardiol 2006; 48:193-202.

15. TAKANO M, YAMAMOTO M, MIZUNO K. Two cases of coronary stent thrombosis very late after bare - metal stenting. J Am Coll Cardiol Intv.2009;2:1286-7.

16. MEHTA S, YUSUF S, PETERS R. Effects of pretreatment with clopidogrel and aspirina followed by long-term therapy in patients undergoing percutaneous coronary intervention: the PCI - CURE study. Lancet.2001;358:527-33.

17. GRINES CL, BONOW RO, CASEY DE JR, GARDNER TJ, LOCKHART PB, MOLITERNO DJ, et al. Prevention of premature discontinuation of dual antiplatelet therapy in patients with coronary artery stents: a science advisory from the American Heart Association, American College of Cardiology, Society for Cardiovascular Angiography and Interventions, American College of Surgeons , and AmericannDental Association , with representation from the American College of Physicians . J Am Coll Cardiol.2007;49:734-9.

18. EISENSTEIN EL, ANSTROM KJ, KONG DF, SHAW LK, TUTTLE RH, MARK DB, et al. Clopidogrel use and long-term clinical outcomes after drug-eluting stent implantation. JAMA. 2007;297:159-68.

19. BRAR SS, KIM J, BRAR SK, ZADEGAN R, REE M, LIU IL, et al. Long -term outcomes by clopidogrel duration and stent type in a diabetic population with de novo coronary artery lesions. J Am Coll Cardiol.2008;51:2220-7 . 